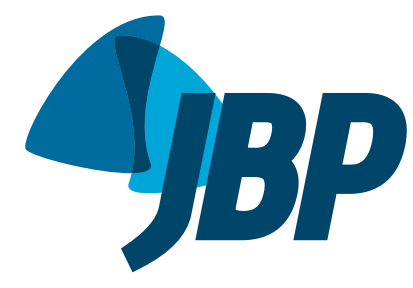

\title{
Bronchoscopic diagnostic procedures and microbiological examinations in proving endobronchial tuberculosis
}

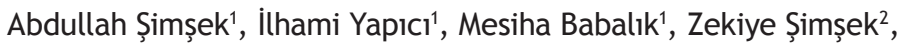 \\ Mustafa Kolsuz ${ }^{1}$
}

1. Department of Chest Diseases, Prof. Dr. Türkan Akyol Chest Diseases Hospital, Bursa, Turkey

2. Department of Radiology, Bursa Çekirge Public Hospital, Bursa, Turkey.

Submitted: 13 June 2015. Accepted: 14 March 2016.

Study carried out in the Department of Chest Diseases, Prof. Dr. Türkan Akyol Chest Diseases Hospital, and in the Department of Radiology, Bursa Çekirge Public Hospital, Bursa, Turkey.

\begin{abstract}
Objective: To determine the proportional distribution of endobronchial tuberculosis (EBTB) subtypes and to evaluate the types of bronchoscopic diagnostic procedures that can prove granulomatous inflammation. Methods: This was a retrospective study of 18 HIV-negative patients with biopsy-proven EBTB treated between 2010 and 2014. Results: The most common EBTB subtypes, as classified by the bronchoscopic features, were tumorous and granular (in 22.2\% for both). Sputum smear microscopy was performed in 11 patients and was positive for AFB in 4 (36.3\%). Sputum culture was also performed in 11 patients and was positive for Mycobacterium tuberculosis in 10 (90.9\%). Smear microscopy of BAL fluid (BALF) was performed in 16 patients and was positive for AFB in $10(62.5 \%)$. Culture of BALF was also performed in 16 patients and was positive for $M$. tuberculosis in 15 (93.7\%). Culture of BALF was positive for M. tuberculosis in $93.7 \%$ of the 16 patients tested. Among the 18 patients with EBTB, granulomatous inflammation was proven by the following bronchoscopic diagnostic procedures: bronchial mucosal biopsy, in 8 (44.4\%); bronchial brushing, in 7 (38.8\%); fine-needle aspiration biopsy, in 2 (11.1\%); and BAL, in 2 (11.1\%). Bronchial anthracofibrosis was observed in $5(27.7 \%)$ of the 18 cases evaluated. Conclusions: In our sample of EBTB patients, the most common subtypes were the tumorous and granular subtypes. We recommend that sputum samples and BALF samples be evaluated by smear microscopy for AFB and by culture for $M$. tuberculosis, which could increase the rates of early diagnosis of EBTB. We also recommend that bronchial brushing be employed together with other bronchoscopic diagnostic procedures in patients suspected of having EBTB.
\end{abstract}

Keywords: Tuberculosis, pulmonary; Mycobacterium tuberculosis; Diagnostic techniques and procedures; Bronchoscopy.

\section{INTRODUCTION}

Pulmonary tuberculosis is one of the major health problems worldwide. There has recently been a resurgence of pulmonary tuberculosis, and that resurgence is related to the HIV epidemic, the emergence of multidrug-resistant strains of Mycobacterium tuberculosis, poverty, and immigration, as well as to a lack of resources in the prevention and treatment system. ${ }^{(1-3)}$ Endobronchial tuberculosis (EBTB) is defined as tuberculosis of the tracheobronchial tree with microbial and histopathological evidence, with or without parenchymal involvement. ${ }^{(4)}$ EBTB is a special form of pulmonary tuberculosis. Previous studies have reported that $10-40 \%$ of patients with active pulmonary tuberculosis have EBTB. ${ }^{(5,6)}$ EBTB can mimic a variety of pulmonary diseases such as bronchogenic carcinoma, pneumonia, and bronchial asthma. The diagnosis of typical pulmonary tuberculosis is easily confirmed by bacteriological means and on the basis of radiological findings. However, EBTB is more difficult to diagnose because of its variable clinical manifestations. Chung et al. ${ }^{(7)}$ divided EBTB into seven subtypes according to the features observed during bronchoscopy: actively caseating, fibrostenotic, edematous-hyperemic, tumorous, ulcerative, granular, and nonspecific. Other authors have found that classification system to be valuable in predicting the therapeutic outcome of EBTB. ${ }^{(8)}$ In the present study, we aimed to determine the proportional distribution of the EBTB subtypes, to evaluate the types of bronchoscopic diagnostic procedures that can prove granulomatous inflammation, and to compare bronchoscopic features with positivity for M. tuberculosis in BAL fluid and sputum samples. Thus, we wanted to show what kinds of procedures are especially needed in order to prove granulomatous inflammation and to define the relationship between bronchoscopic features and positivity for M. tuberculosis.

\section{METHODS}

The Türkan Akyol Chest Diseases Public Hospital, in the city of Bursa, Turkey, is one of several referral 
hospitals for tuberculosis in the country. This was a retrospective study of 18 HIV-negative patients with biopsy-proven EBTB treated at the hospital between 2010 and 2014. The diagnosis of EBTB was confirmed histopathologically in all 18 of the patients. Some patients were initially unable to expectorate sputum and others were sputum smear-negative according to AFB staining (sputum induction tests with hypertonic saline were not used at our hospital during the study period). Fiberoptic bronchoscopy was performed in the case of suspected tuberculosis or for the differentia diagnosis of tuberculosis. Some patients expectorated sputum after fiberoptic bronchoscopy. A flexible bronchoscope was inserted through the nasal passage. Forceps were advanced through the bronchoscope and airway to obtain biopsies from bronchial lesions. The characteristics of the patients, including demographic data, as well as radiological, bronchoscopic, and microbiological features, were reviewed, evaluated, and recorded retrospectively, as were the types of bronchoscopic diagnostic procedures employed in order to prove granulomatous inflammation, such as bronchial mucosal biopsy, fine-needle aspiration biopsy, bronchial brushing, and BAL. Bronchoscopic findings were categorized according to the classification system devised by Chung et al.(7) Bronchial anthracofibrosis was also recorded as a bronchoscopic finding. The results are presented as means \pm standard deviations or as absolute and relative frequencies.

\section{RESULTS}

Between 2010 and 2014, a total of 1,380 patients were diagnosed with pulmonary tuberculosis at our hospital. Among those 1,380 patients, endobronchial lesions were observed in 34 (2.46\%), of whom 18 (52.9\%) were histopathologically diagnosed with EBTB. During the study period, 3,325 patients had been examined with fiberoptic bronchoscopy because of suspicion of pulmonary tuberculosis. Among the patients with EBTB, the female-to-male ratio was $1.57: 1$; ages ranged from 16 to 83 years; the mean age was $53.1 \pm 20.1$ years; and $38.8 \%$ of the patients were under 45 years of age.

Anatomically, the bronchoscopic findings were located primarily in the right upper lobe bronchus, in $5(27.8 \%)$ of the 18 patients with EBTB, followed by the right lower lobe bronchus, in 4 (22.2\%). Bilateral pulmonary involvement was observed in 5 patients $(27.8 \%)$, right middle lobe involvement was observed in $1(5.6 \%)$, right middle/upper lobe involvement was observed in 1 (5.6\%), left upper lobe involvement was observed in $1(5.6 \%)$, and left main bronchus involvement was observed in 1 (5.6\%). Bronchoscopic features, as classified with the Chung et al. system, ${ }^{(7)}$ are listed in Table 1.

Radiologic alterations were observed in 14 patients: heterogeneous infiltration in 7 cases (50\%); nodular infiltration in 7 cases (50\%); ground-glass appearance in 5 cases (35.7\%); consolidation in 5 cases ( $35.7 \%$ ); atelectasis in 5 cases (35.7\%); mass lesion in 4 cases $(27.5 \%)$; lymphadenopathy in 3 cases $(21.4 \%)$; pleural effusions in 2 cases (14.3\%); and cavitary infiltration in 1 case $(7.1 \%)$. Middle lobe syndrome was seen in 1 case $(7.1 \%)$, and miliary tuberculosis was seen in 2 cases (14.3\%). The lesions were mostly unilateral, being found in the right lung in 11 cases (78.5\%). Multilobar involvement was observed in 9 cases $(64.3 \%)$.

Table 2 shows the results of the microbiological and smear examinations of sputum samples evaluated in 11 cases. In some cases, the patients were unable to produce sputum, and some sputum samples were collected after fiberoptic bronchoscopy. Of the 11 samples submitted to staining, 4 (36.3\%) were positive for AFB. The highest smear positivity for AFB (100\%) was found in the patients with tumorous EBTB. None of the patients with edematous-hyperemic EBTB or nonspecific EBTB were sputum smear-positive for AFB. Ten $(90.9 \%)$ of the 11 patients had a positive sputum culture for M. tuberculosis, and the remaining patient had nonspecific EBTB.

In two cases (one case of caseating EBTB and one case of nonspecific EBTB), the BAL fluid had not been sent for AFB staining. Therefore, microbiological and smear examinations of BAL fluid for $M$. tuberculosis were evaluated in only 16 patients (Table 3 ). Of those 16 patients, $10(62.5 \%)$ were positive for AFB from BAL. The highest BAL smear positivity for AFB (100\%) was found in the patients with tumorous EBTB. The BAL fluid culture was positive for $M$. tuberculosis in $15(93.7 \%)$ of the patients, and the remaining patient had fibrostenotic EBTB.

Among the 18 patients with EBTB, granulomatous inflammation was proven by the following bronchoscopic diagnostic procedures (Table 4): bronchial mucosal biopsy, in 8 cases (44.4\%); bronchial brushing, in 7 cases $(38.8 \%)$; fine-needle aspiration biopsy, in 2 cases (11.1\%); and $B A L$, in 2 cases (11.1\%). In one case of nonspecific EBTB, fine-needle aspiration biopsy and bronchial brushing both revealed granulomatous inflammation. Bronchial anthracofibrosis was identified in 5 cases (27.7\%): in 2 patients with tumorous EBTB (11.1\%); in 2 with granular EBTB (11.1\%); and in 1 with caseating EBTB (5.5\%).

Table 1. Classification of endobronchial tuberculosis, by bronchoscopic features. ${ }^{a}$

\begin{tabular}{|c|c|}
\hline EBTB subtype ${ }^{b}$ & $(n=18)$ \\
\hline Tumorous & $4(22.2)$ \\
\hline Granular & $4(22.2)$ \\
\hline Caseating & $3(16.7)$ \\
\hline Edematous-hyperemic & $3(16.7)$ \\
\hline Nonspecific & $2(11.1)$ \\
\hline Fibrostenotic & $1(5.6)$ \\
\hline Ulcerative & $1(5.6)$ \\
\hline
\end{tabular}


Table 2. Results of microbiological examination of sputum, by endobronchial tuberculosis subtype. ${ }^{a}$

\begin{tabular}{|c|c|c|c|}
\hline EBTB subtype ${ }^{b}$ & $\mathbf{n}$ & Sputum smear positivity for AFB & $\begin{array}{c}\text { Culture positivity for } \\
\text { Mycobacterium tuberculosis }\end{array}$ \\
\hline Tumorous & 2 & $2(100.0)$ & $2(100.0)$ \\
\hline Granular & 3 & $1(33.3)$ & $3(100.0)$ \\
\hline Caseating & 2 & $1(50.0)$ & $2(100.0)$ \\
\hline Edematous-hyperemic & 2 & $0(0)$ & $2(100.0)$ \\
\hline Nonspecific & 2 & $0(0)$ & $1(50.0)$ \\
\hline Fibrostenotic & - & & \\
\hline Ulcerative & - & & \\
\hline Total & 11 & $4(36.3)$ & $10(90.9)$ \\
\hline
\end{tabular}

Table 3. Results of microbiological examination of BAL fluid, by endobronchial tuberculosis subtype. ${ }^{\text {a }}$

\begin{tabular}{llcc}
\multicolumn{1}{c}{ EBTB subtype ${ }^{b}$} & $\mathbf{n}$ & Smear positivity for AFB & $\begin{array}{c}\text { Culture positivity for } \\
\text { Mycobacterium tuberculosis }\end{array}$ \\
Tumorous & 4 & $4(100.0)$ & $4(100.0)$ \\
Granular & 4 & $2(50.0)$ & $4(100.0)$ \\
Caseating & 2 & $1(50.0)$ & $2(100.0)$ \\
Edematous-hyperemic & 3 & $2(66.9)$ & $3(100.0)$ \\
Nonspecific & 1 & $0(0)$ & $1(100.0)$ \\
Fibrostenotic & 1 & $0(0)$ & $0(0)$ \\
Ulcerative & 1 & $1(100.0)$ & $1(100.0)$ \\
Total & 16 & $10(62.5)$ & $15(93.7)$ \\
\hline
\end{tabular}

EBTB: endobronchial tuberculosis. aResults expressed as $\mathrm{n}(\%) .{ }^{\mathrm{b}}$ Classification system devised by Chung et al.(7)

Table 4. Detection of granulomatous inflammation, by bronchoscopic diagnostic procedure employed. ${ }^{a}$

\begin{tabular}{|c|c|c|c|c|}
\hline EBTB subtype ${ }^{b}$ & BAL & $\begin{array}{l}\text { Bronchial mucosal } \\
\text { biopsy }\end{array}$ & $\begin{array}{c}\text { Fine-needle } \\
\text { aspiration biopsy }\end{array}$ & Bronchial brushing \\
\hline Tumorous & & 4 & & \\
\hline Granular & & 3 & & 1 \\
\hline Caseating & & 1 & & 2 \\
\hline Edematous-hyperemic & 1 & & 1 & 1 \\
\hline Nonspecific & 1 & & 1 & 1 \\
\hline Fibrostenotic & & & & 1 \\
\hline Ulcerative & & & & 1 \\
\hline Total & 2 & 8 & 2 & 7 \\
\hline
\end{tabular}

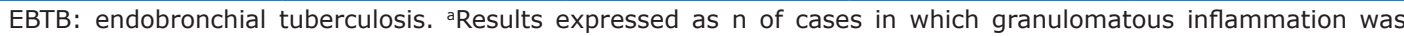

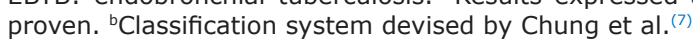

\section{DISCUSSION}

In present study, 18 patients were histopathologically diagnosed with EBTB. The lesions were mostly unilateral (in the right lung) and multilobar. The most common radiological findings were heterogeneous infiltration (in 50\%) and nodular infiltration (in 50\%). The most common EBTB subtypes were the tumorous subtype (in $22.2 \%$ ) and the granular subtype (in $22.2 \%$ ). Sputum smear microscopy was positive for AFB in $36.3 \%$ of the patients. The BAL fluid was positive for AFB in $62.5 \%$ of the patients. Among the diagnostic procedures employed in order to prove granulomatous inflammation, bronchial mucosal biopsy and bronchial brushing were the most effective. Bronchial anthracofibrosis was found in $27.7 \%$ of the cases.

Although the reasons are unclear, EBTB is more often observed in female patients. Possible explanations for that include the fact that females do not expectorate sputum as well as do males, because women have thinner bronchial lumina, as well as because there are sociocultural and aesthetic proscriptions against women expectorating. In our sample of patients with EBTB, the female-to-male ratio was $1.57: 1$, which is consistent with the preponderance of females reported in other studies of EBTB. ${ }^{(8-11)}$ EBTB usually affects adults, although younger and elderly patients can be affected, EBTB patient ages ranging from 14 to 81 years. ${ }^{(12)}$ In the present study, patients ranged from 16 to 83 years of age. The mean age was 53.1 years, and $38.8 \%$ of the patients were under 45 years of age, which is also in keeping with data in the literature. ${ }^{(8-11)}$

In the present study, the most common EBTB subtype was actively caseating EBTB, as has been reported in some previous studies. ${ }^{(8,11)}$ However, in a study conducted by Qingliang et al., ${ }^{(10)}$ the granular 
subtype was the most common (in $31.8 \%$ of the patients), whereas the edematous-hyperemic type was the most common (in $34.7 \%$ of the patients) in a study conducted by Ozkaya et al. ${ }^{(9)}$ In our study, the tumorous and granular subtypes were both seen in $22.2 \%$ of the patients, which differs from that reported in other studies. ${ }^{(10)}$ The ulcerative and fibrostenotic subtypes were the least common in our study, both being seen in $5.6 \%$ of the patients.

The yield of sputum smear microscopy for AFB is not as high in EBTB as it is in parenchymal involvement, even in an optimal laboratory set up for meticulous sputum examination. In recent studies, sputum positivity in EBTB has been demonstrated to range from $16.0 \%$ to $53.3 \%$. ${ }^{(7,13,14)}$ In an even more recent study, ${ }^{(9)}$ sputum smear microscopy for AFB was negative in all patients. In the present study, sputum smear microscopy for AFB was positive in $36.3 \%$ of the patients and the M. tuberculosis culture positivity rate was very high $(90.9 \%)$. Therefore it can be said that, when it is possible to collect sputum samples (before or after bronchoscopy), it is worthwhile to send those samples for microbiological examination for AFB.

Direct sputum smear microscopy remains a fundamental tool in the diagnosis of tuberculosis. Alternative methods of obtaining sputum specimens, including sputum induction, BAL, and gastric lavage, are frequently called for in patients with radiological suspicion of tuberculosis who are unable to expectorate or are smear-negative. In a study conducted by McWilliams et al., ${ }^{(15)}$ the yield of induced sputum (96.3\%) was superior to that of BAL fluid (51.9\%) and the overall cost of BAL was found to be three times that of performing sputum induction. In comparison with BAL, sputum induction has several advantages ${ }^{(16)}$ : it is less invasive; it has a higher diagnostic yield; it provides greater patient comfort and safety; it is a low-cost procedure; there is no age restriction on its use; it does not require patient fasting; it is an outpatient procedure; it can be performed without the involvement of an expert; and it is less time-consuming. Unfortunately, sputum induction was not employed at our hospital during the study period.

The second step in the clinical evaluation of EBTB is bronchoscopy, in order to examine bronchial structures and obtain specimens for diagnosis. In the study conducted by Ozkaya et al., ${ }^{(9)}$ the BAL fluid was positive for AFB in $26.0 \%$ of the cases and the BAL fluid culture was positive for M. tuberculosis in $39.1 \%$. The authors found that positivity for AFB was highest (75.0\%) among the patients with the granular subtype of EBTB. They also found mycobacterial culture positivity to be highest (also $75.0 \%$ ) among the patients with the granular subtype. ${ }^{(9)}$ In our study, microbiological and smear examinations of BAL fluid for AFB were both positive in $62.5 \%$ of the patients. Cultures of BAL fluid for M. tuberculosis were positive in $93.7 \%$ of our patients. According to our data, microbiological and smear examinations of BAL fluid have high rates of positivity of staining for AFB and culture for $M$. tuberculosis. It is therefore worthwhile to send BAL fluid samples for AFB analysis in order to facilitate the early diagnosis of EBTB.

Various bronchoscopic specimens, including those obtained through biopsy, bronchial brushing, or BAL, can be evaluated. ${ }^{(12)} \mathrm{A}$ bronchoscopic biopsy is the most reliable method for diagnosing EBTB, because a needle aspiration biopsy sample can provide only a cytological diagnosis. The reported rate of positivity in bronchial biopsy samples ranges from $30 \%$ to $84 \% .^{(13,17)}$ We found it surprising that, in our study, the diagnostic yield of bronchial brushing was nearly equal to that of bronchial mucosal biopsy in detecting granulomas (38.8\% and $44.4 \%$, respectively). In a clinical analysis of 90 cases of EBTB in China, (13) bronchial brushing yielded variable results, ranging from $10 \%$ to $85 \%$. In the present study, bronchial mucosal biopsy was especially effective in diagnosing EBTB in patients with the tumorous or granular subtypes (positivity rate of $100 \%$ and $75 \%$, respectively), bronchial brushing proving diagnostic in patients with any of the other subtypes.

Tuberculosis is one of the most common diseases associated with bronchial anthracofibrosis. ${ }^{(18,19)}$ Bronchial anthracofibrosis is typically induced by the long-term inhalation of biomass smoke. ${ }^{(18)}$ Previous studies have reported high rates of tuberculosis in patients with bronchial anthracofibrosis. ${ }^{(18,20-25)}$ In the present study, bronchial anthracofibrosis was found in 5 cases $(27.7 \%)$ : in 2 patients with tumorous EBTB (11.1\%); in 2 with granular EBTB (11.1\%); and in 1 with caseating EBTB (5.5\%). These findings differ from those reported by Kim et al.,(26) who found actively caseating, edematous-hyperemic, and ulcerative EBTB to be the most common EBTB subtypes, respectively occurring in $49 \%, 21 \%$, and $20 \%$ of their patients.

The results of the present study show the value of staining for AFB and culture for M. tuberculosis in sputum and BAL fluid samples for the early diagnosis of EBTB. In addition, because of its high diagnostic power, we can state that bronchial brushing is a recommended bronchoscopic diagnostic procedure in patients with suspected EBTB.

Our study has certain limitations. Primarily, due to the retrospective nature of the study, we relied on electronic medical records as our source of patient data.

In conclusion, the tumorous and granular subtypes were the EBTB subtypes most commonly seen in our study. Because of the high positivity rates, we recommend that sputum samples and BAL fluid samples be evaluated by smear microscopy for AFB and by culture for M. tuberculosis, which could collectively increase the rates of early diagnosis of EBTB. On the basis of our findings, we also recommend that bronchial brushing be employed together with other bronchoscopic diagnostic procedures, in order to increase the diagnostic yield, in patients suspected of having EBTB. 


\section{REFERENCES}

1. Millard PS, Cegielski JP, Wing S, Silver A. Rurality and tuberculosis incidence trends in North and South Carolina, 1980 to 1992. J Rural Health. 1994;10(4): 226-36. http://dx.doi. org/10.1111/j.1748-0361.1994.tb00236.x

2. Glynn JR. Resurgence of tuberculosis and the impact of HIV infection. Br Med Bull. 1998;54(3):579-93. http://dx.doi.org/10.1093/ oxfordjournals.bmb.a011712

3. Lerner $\mathrm{BH}$. Catching patients: tuberculosis and detention in the 1990s. Chest. 1999;115(1):236-41. http://dx.doi.org/10.1378/ chest.115.1.236

4. Lee JH, Park SS, Lee DH, Shin DH, Yang SC, Yoo BM. Endobronchial tuberculosis. Clinical and bronchoscopic feature in 121 cases. Chest. 1992;102(4): 990-4. http://dx.doi.org/10.1378/chest.102.4.990

5. Calpe JL, Chiner E, Larramendi $\mathrm{CH}$. Endobronchial tuberculosis in HIV-infected patients. AIDS. 1995;9(10):1159-64. http://dx.doi. org/10.1097/00002030-199510000-00007

6. Han JK, Im JG, Park JH, Han MC, Kim YW, Shim YS. Bronchial stenosis due to endobronchial tuberculosis: successful treatment with self-expanding metallic stent. AJR Am J Roentgenol. 1992;159(5):971-2. http://dx.doi.org/10.2214/ajr.159.5.1414809

7. Chung HS, Lee JH, Han SK, Shim YS, Kim KY, Han YC, et al. Classification of endobronchial tuberculosis by the bronchoscopic features. Tuberc Respir Dis. 1991;38:108-15.

8. Chung HS, Lee JH. Bronchoscopic assessment of the evolution of endobronchial tuberculosis. Chest. 2000;117(2):385-92. http://dx.doi. org/10.1378/chest.117.2.385

9. Ozkaya S, Bilgin S, Findik S, Kök HC, Yuksel C, Atıcı AG. Endobronchial tuberculosis: histopathological subsets and microbiological results. Multidiscip Respir Med. 2012;7(1):34. http://dx.doi.org/10.1186/20496958-7-34

10. Qingliang $X$, Jianxin W. Investigation of endobronchial tuberculosis diagnoses in 22 cases. Eur J Med Res. 2010;15:309-13. http://dx.doi. org/10.1186/2047-783X-15-7-309

11. Sahin F, Yıldız P. Characteristics of endobronchial tuberculosis patients with negative sputum acid-fast bacillus. J Thorac Dis. 2013;5(6):764-70.

12. Kashyap S, Mohapatra PR, Saini V. Endobronchial tuberculosis. Indian J Chest Dis Allied Sci. 2003;45(4):247-56.

13. Yu W, Rong Z. Clinical analysis of 90 cases with endobronchial tuberculosis [Article in Chinese]. Zhonghua Jie He He Hu Xi Za Zhi. 1999;22(27):396-8.

14. Aggarwal AN, Gupta D, Joshi K, Behera D, Jindal SK. Endobronchial involvement in tuberculosis: a report of 24 cases diagnosed by fibreoptic bronchoscopy. J Bronchol. 1999;6:247-50. http://dx.doi. org/10.1097/00128594-199910000-00004

15. McWilliams T, Wells AU, Harrison AC, Lindstrom S, Cameron RJ Foskin E. Induced sputum and bronchoscopy in the diagnosis of pulmonary tuberculosis. Thorax. 2002;57(12):1010-4. http://dx.doi. org/10.1136/thorax.57.12.1010

16. Anderson C, Inhaber N, Menzies D. Comparison of sputum induction with fiber-optic bronchoscopy in the diagnosis of tuberculosis. Am J Respir Crit Care Med. 1995;1525 Pt 1):1570-4.

17. Altin S, Cikrikçioğlu S, Morgül M, Koşar F, Ozyurt H. 50 endobronchial tuberculosis cases based on bronchoscopic diagnosis. Respiration. 1997;64(2):162-4. http://dx.doi.org/10.1159/000196662

18. Kim YJ, Jung CY, Shin HW, Lee BK. Biomass smoke induced bronchial anthracofibrosis: presenting features and clinical course. Respir Med. 2009;103(5):757-65. http://dx.doi.org/10.1016/1. rmed.2008.11.011

19. Hwang J, Puttagunta L, Green F, Shimanovsky A, Barrie J, Long R. Bronchial anthracofibrosis and tuberculosis in immigrants to Canada from the Indian subcontinent. Int J Tuberc Lung Dis. 2010;14(2):2317.

20. Chung MP, Lee KS, Han J, Kim H, Rhee $\mathrm{CH}$, Han YC, et al. Bronchia stenosis due to anthracofibrosis. Chest. 1998;113(2):344-50. http:// dx.doi.org/10.1378/chest.113.2.344

21. Mirsadraee $M$, Saeedi $P$. Anthracosis of lung: evaluation of potentia underlying causes. J Bronchol. 2005;12(2):84-7. http://dx.doi. org/10.1097/01.laboratory.0000150873.99404.53

22. Pérez-Padilla R, Pérez-Guzmán C, Báez-Salda-a R, Torres-Cruz A Cooking with biomass stoves and tuberculosis: a case control study. Int J Tuberc Lung Dis. 2001;5(5):441-7.

23. Pokhrel AK, Bates MN, Verma SC, Joshi HS, Sreeramareddy $\mathrm{CT}$, Smith KR. Tuberculosis and indoor biomass and kerosene use in Nepal: a case-control study. Environ Health Perspect. 2010;118(4):558-64. http://dx.doi.org/10.1289/ehp.0901032

24. Kolappan C, Subramani R. Association between biomass fue and pulmonary tuberculosis: a nested case-control study. Thorax. 2009;64(8):705-8. http://dx.doi.org/10.1136/thx.2008.109405

25. Rajagopalan S. Tuberculosis and aging: a global health problem. Clin Infect Dis. 2001;33(7):1034-9. http://dx.doi.org/10.1086/322671

26. Kim HJ, Kim SD, Shin DW, Bae SH, Kim AL, Kim JN, et al. Relationship between bronchial anthracofibrosis and endobronchial tuberculosis. Korean J Intern Med. 2013;28(3):330-8. http://dx.doi. org/10.3904/kjim.2013.28.3.330 